\title{
Investigating Cytokine Binding Using a Previously Reported TNF-Specific Aptamer
}

\author{
James D. Fisher ${ }^{1,2}$, Morgan V. DiLeo ${ }^{1,3}$, William J. Federspiel ${ }^{1,2,3,4^{*}}$ \\ ${ }^{1}$ McGowan Institute for Regenerative Medicine, University of Pittsburgh, Pittsburgh, USA \\ ${ }^{2}$ Departments of Chemical Engineering, University of Pittsburgh, Pittsburgh, USA \\ ${ }^{3}$ Departments of Bioengineering, University of Pittsburgh, Pittsburgh, USA \\ ${ }^{4}$ Departments of Surgery, University of Pittsburgh, Pittsburgh, USA \\ Email: fisherj4@upmc.edu,mod8@pitt.edu, federspielwj@upmc.edu
}

Received May 27, 2012; revised June 28, 2012; accepted July 14, 2012

\begin{abstract}
Cytokines are of chief importance in the pathophysiology of sepsis and other systemic inflammatory response syndromes. We are designing and testing an extracorporeal cytokine adsorption device (CAD) that can remove cytokines via adsorption on biocompatible, microporous beads. The goal of this study was to determine whether a previously reported TNF binding DNA aptamer, 5'-GCGGCCGATA AGGTCTTTCC AAGCGAACGA ATTGAACCGC-3', could be immobilized our hemoadsorption polymer surface to increase the removal rate of TNF. A reservoir consisting of horse serum spiked with a known concentration of TNF was perfused through our CAD packed with aptamer modified or unmodified (control) polymer beads. The binding affinity of the TNF aptamer was characterized using an enzyme-linked oligonucleotide assay (ELONA). As a positive control a well-established DNA aptamer that binds PDGF $\mathrm{BB}$ was also subjected to the same ELONA to validate the assay. TNF capture using the CAD showed no TNF removal over four hours for both the aptamer modified and unmodified control beads. Additionally, the results of the ELONA showed no binding of TNF to the reported aptamer; however the PDGF BB aptamer did bind PDGF BB. Based on these results we are able to conclude that the reported TNF specific aptamer does not bind TNF. These results will be of importance to other studies exploring aptamers for specific binding of TNF.
\end{abstract}

Keywords: Aptamers; Sepsis; Hemoadsorption; TNF

\section{Introduction}

Sepsis is defined as systemic inflammation in the presence of infection. The worldwide prevalence of this disease and the lack of efficient treatment options have made sepsis one of the leading causes of death in the world and the most common cause of death in adult, noncoronary intensive care units [1]. Systemic inflammation results in the excessive production of pro-inflammatory cytokines such as tumor necrosis factor (TNF) and interleukin-6 (IL-6) and anti-inflammatory cytokines such as interleukin-10 (IL-10). Overproduction of cytokines along with their interactions with one another contribute to the pathological process of sepsis [2].

One emerging therapy aimed at treating sepsis is hemofiltration. The aim of this therapy is to remove proand anti-inflammatory cytokines from circulation using convection, thus reestablishing a physiological balance. Hemofiltration studies with sepsis have shown an initial decrease in cytokine removal followed by a gradual decrease as the filter becomes saturated [3]. However, Kel-

\footnotetext{
${ }^{*}$ Corresponding author.
}

lum and Dishart (2002) were able to show evidence that the primary mechanism responsible for interleukin-6 (IL-6) removal during hemofiltration is most likely due to adsorption onto the membrane rather than filtration from plasma [4]. Thus, newer therapies now focus on hemoadsorption in addition to hemofiltration. Our group is developing a cytokine capture device that consists of a column packed with microporous polymer beads through which whole blood is perfused [5]. The polymer beads used in the cytokine adsorption device (CAD) have a high surface area $\left(850 \mathrm{~m}^{2} / \mathrm{g}\right)$, making them ideal for adsorption-based therapy.

Our device removes a variety of middle-molecular weight proteins in the $10-30 \mathrm{kDa}$ range including cytokines generally considered of clinical relevance to sepsis such as IL-6, IL-10, and TNF. While our device has been effective at removing both IL- 6 and IL-10 in both in vitro and ex vivo animal studies, the removal rate of TNF has been considerably slower than that of IL-6 and IL-10 [6]. To address this issue, we have begun studying specific capture of TNF by immobilized ligands on the outer surface of the polymer beads currently used in the CAD as a 
means to increase the removal rate of TNF. An ongoing focus in our group has been immobilizing antibodies as specific capture ligands for TNF. Antibodies have several drawbacks, including the substantial cost associated with coating several grams of polymer beads with TNF antibodies, in addition to their limited shelf life. Another concern is the potentially harmful immune response that may occur if antibodies leach off of the beads.

A novel alternative to antibodies are aptamers, short strands of oligonucleotides. Aptamers fold into a unique three dimensional structure (similar to antibodies) which allows them to specifically bind to a variety of biomolecules with affinity constants comparable to that of antibodies $\left(\sim 10^{-9} \mathrm{M}\right)$. These nucleic acid oligomers are synthesized via an iterative in vitro selection process called SELEX (systematic evolution of ligands by exponential enrichment) [7,8]. Aptamers possess several advantages over their antibody counterparts as they are less expensive, synthesized in vitro, have longer shelf lives and are less likely to elicit immunogenicity than antibodies $[9,10]$. Moreover, various chemical functionalities can be added to the 5' and 3 ' ends of the aptamer to allow for easier conjugation to a surface.

In this work, our group investigated the TNF specific aptamer sequence, 5'-GCGGCCGATA AGGTCTTTCC AAGCGAACGA ATTGAACCGC-3', reported in the patent submitted by Zhang et al. [11]. We immobilized this aptamer on the surface of porelesspolystyrene-divinylbenzene (PSDVB) beads and tested its ability to bind TNF in the CAD and subsequently deplete it from the circulating serum solution. Poreless PSDVB beads were used since the aptamers, unlike antibodies, are small enough to diffuse into the porous network of our standard porous PSDVB beads. In addition, the specificity of this aptamer sequence to human TNF alpha was evaluated using the enzyme-linked oligonucleotide assay (ELONA) methodologies of Yan et al. [12].

\section{Methods}

Carboxyl groups were incorporated onto the surface of the poreless PSDVB beads to provide a functionalized surface for aptamer coupling, using a modified polystyrene oxidation protocol [13]. Batches of 2 grams of poreless PSDVB beads were incubated in manganese (VII) oxide in $\mathrm{H}_{2} \mathrm{SO}_{4}$ at $65^{\circ} \mathrm{C}$ for 1 hour. The surface concentration of carboxyl groups was measured to be $24 \mathrm{nmol} / \mathrm{g}$ polymer using a para-nitrophenol colorimetric assay [14]. Beads were washed with $6 \mathrm{~N}$ hydrochloric acid and DI water. Functional groups were activated by incubating the beads with a $1 \mathrm{mg} / \mathrm{ml}$ solution of 1-ethyl-3-(3-dimethylaminopropyl) carbodiimide (EDC) in 2-(N-morpholino) ethanesulfonic acid (MES) buffer ( $\mathrm{pH} 4.5$ ) for 1 hour. The beads were washed with MES buffer followed by DI water. Aptamers were coupled to the beads by adding 11 $\mu \mathrm{g} / \mathrm{ml}$ aptamer solution in sodium phosphate buffer $(\mathrm{pH}$
7.0) and incubating at room temperature for 2 hours. Aptamers used in this step were functionalized with an amine group at the 5' end for coupling. Beads were washed with a $.05 \%$ Tween solution followed by DI water.

For TNF capture experiments, 1.5 grams of aptamerimmobilized beads or unmodified beads were packed in an unused $\mathrm{CAD}$ and connected in series with a peristaltic pump. Inlet and outlet tubes were connected to an $8 \mathrm{ml}$ reservoir of horse serum spiked with TNF at a concentration of $\sim 1200 \mathrm{pg} / \mathrm{ml}$. The reservoir was perfused through the CAD at a flow rate of $0.8 \mathrm{ml} / \mathrm{min}$ and samples were taken at $t=0,15,30,60,90,120,180,240 \mathrm{~min}$. TNF concentrations were measured using a Biosource enzyme-linked immunosorbent assay (ELISA) (Invitrogen) according to the manufacturer's instructions.

The ELONA technique was performed as follows. Recombinant human TNF (ThermoFisher) was diluted to $5 \mu \mathrm{g} / \mathrm{ml}$ using coating buffer ( $0.05 \mathrm{M}$ Sodium Carbonate $\mathrm{pH}$ 9.76), and $100 \mu \mathrm{l}$ of this solution was incubated overnight at $4^{\circ} \mathrm{C}$ in a polystyrene microplate. As a negative control, recombinant human IL-6 (Thermo) at the same concentration was used. The plate was washed with a $0.15 \mathrm{M} \mathrm{NaCl}$ buffer, $\mathrm{pH} 7.4$, containing $0.1 \%$ Tween 20 , and remaining adsorption sites were then blocked with $100 \mu \mathrm{l}$ of $1 \%$ bovine serum albumin (BSA) in phosphatebuffered saline (PBS) for $2 \mathrm{~h}$ at $37^{\circ} \mathrm{C}$. Wells were once again washed followed by addition of $100 \mu \mathrm{l}$ of TNFspecific DNA aptamers at concentrations of $1.10 \times 10^{7}$, $1.10 \times 10^{5}, 1104,552$, and $276 \mathrm{pg} / \mathrm{ml} .100 \mu \mathrm{l}$ of the 1104 $\mathrm{pg} / \mathrm{ml}$ aptamer solution was added to the IL- 6 coated well as a negative control, and $100 \mu \mathrm{l}$ of biotinylated TNF antibody (Biosource) was used a positive control. The aptamer/antibody solutions were incubated at $37^{\circ} \mathrm{C}$ for $1 \mathrm{~h}$ and then washed. $100 \mu \mathrm{l}$ of streptavidin-conjugated horseradish peroxidase (Biosource) was added to each well and incubated at $37^{\circ} \mathrm{C}$ for $1 \mathrm{~h}$. The wells were washed for the final time, after which $100 \mu \mathrm{l}$ of tetramethylbenzidine (TMB) substrate solution was added to each well. After $20 \mathrm{~min}$, the optical density was measured at $450 \mathrm{~nm}$ on a MultiSkan Plus microplate reader (ThermoFisher).

We also evaluated the affinity of a well-established aptamer for its target ligand using ELONA to ensure that the technique was being done correctly. Green et al. published a DNA aptamer sequence, 5'-CAGGCTACGGCACGTAGAGCATCACCATGATCCTG-3', which exhibited high binding affinity towards platelet-derived growth factor BB (PDGF-BB) [15]. The methods used in the PDGF-BB ELONA were the same as those used in the TNF ELONA. Wells were coated with PDGF-BB and the PDGF-BB aptamer was the target analyte.

\section{Results}

Figure 1 shows the results of TNF capture for horse serum perfused through the $\mathrm{CAD}$ packed with aptamer- 
immobilized poreless PSDVB beads and unmodified poreless PSDVB beads (control).

Neither the aptamer-immobilized nor the unmodified beads were able to significantly decrease the circulating concentration of TNF.

The results of the human TNF and PDGF-BB ELONAs are shown in Figures 2 and 3, respectively. The TNF ELONA data indicates that the TNF aptamer exhibited no binding affinity toward recombinant TNF. The positive control, a biotinylated human TNF antibody, showed a significant amount of binding affinity toward TNF rela- tive to the negative control and test wells. The PDGF-BB ELONA, however, demonstrated that the PDGF-BB aptamer did have significant binding affinity to PDGF-BB relative to the control wells. The wells of the PDGF-BB ELONA corresponding to concentrations $4.51 \times 10^{8}$, $4.51 \times 10^{6}$, and $4.5 \times 10^{4} \mathrm{pg} / \mathrm{ml}$ did not show a decrease in signal as these concentrations were beyond the detection limit of the plate reader.

However, the subsequent concentrations showed a decrease in signal with a decrease in concentration of aptamer, ruling out the possibility of non-specific binding.

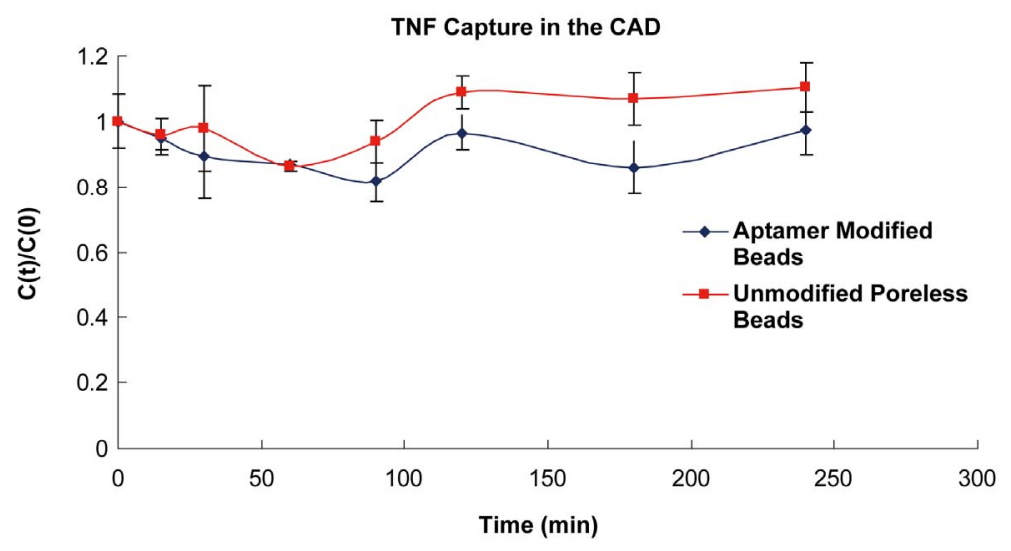

Figure 1. TNF capture with aptamer-immobilized and unmodified PSDVB beads.

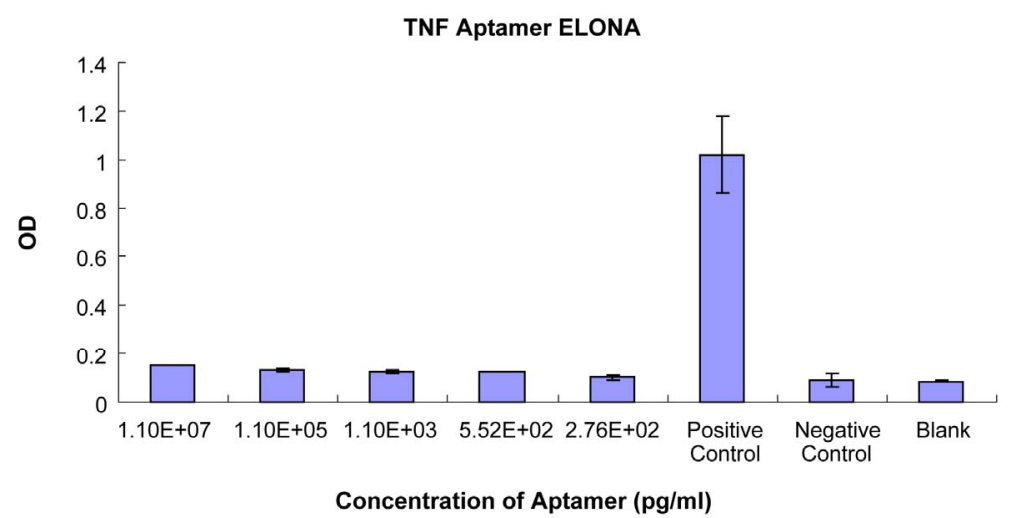

Figure 2. TNF aptamer ELONA.

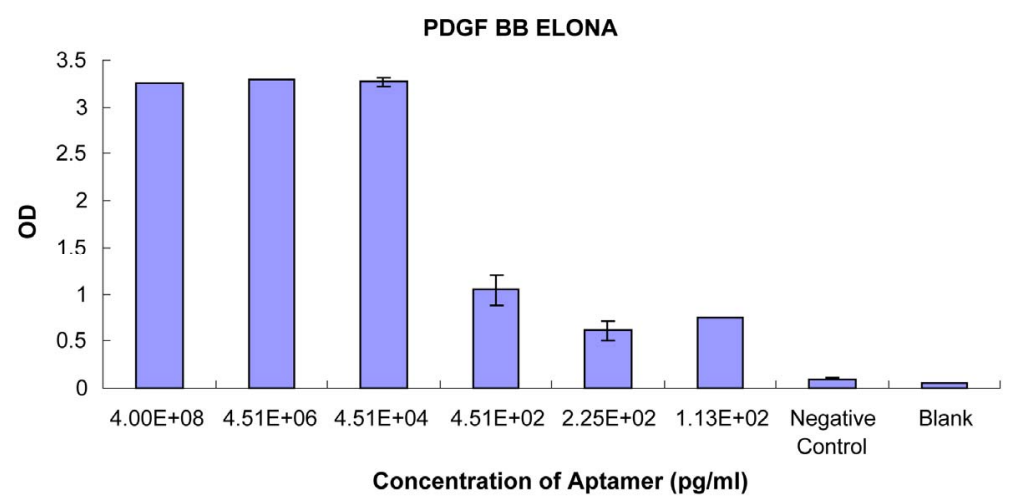

Figure 3. Platelet derived growth factor BB ELONA. 


\section{Discussion}

The ability of CADs packed with aptamer-immobilized or unmodified beads to capture TNF from horse serum was tested. The results in Figure 1 show that TNF capture with unmodified and aptamer-immobilized beads was negligible. The aptamer-immobilized on the surface of the PSDVB beads was reported to specifically bind TNF, therefore we expected that the aptamer-immobilized beads would display a significantly higher ability to capture TNF than the control beads. Based on the surface density of carboxyl groups on the beads, we calculated that if successfully coupled there would be at least a 10 molar excess of aptamer to TNF. Therefore, one possible explanation is that the TNF aptamer was not successfully coupled to the surface of the PSDVB beads. This is unlikely however, as we have successfully coupled antibodies to the PSDVB beads using the same chemistry. Another possible explanation was that the reported aptamer did not bind to TNF. To characterize the affinity of the published aptamer for TNF, we utilized a previously reported enzyme-linked oligonucleotide assay (ELONA) [12].

From the ELONA data we are able to conclude that the TNF aptamer sequence does not specifically bind TNF. There are several possible explanations for this finding. The discrepancies in data could be a result of differences in the protein at which the aptamer was targeted. Our group used commercially available recombinant human TNF from ThermoFisher Scientific, but the recombinant protein used by Zhang et al. was produced in their laboratory. The target proteins were synthesized in different environments, which may suggest that the three dimensional structure of the proteins may have differed enough to impact the aptamer's affinity toward TNF. The TNF used in our work was in its correct threedimensional shape, as evidenced by our positive control, a TNF antibody, being able to bind TNF in the ELONA. A TNF antibody was not used as a positive control in the group's patent or published description of the RNA aptamer $[11,12]$.

The aptamer sequence, 5'-GCGGCCGATA AGGTCTTTCC AAGCGAACGA ATTGAACCGC-3', reported by Zhang and coworkers does not appear to bind commercially available recombinant TNF. While this result is a negative finding, we believe that this correspondence provides important data to other investigators who may be studying specific ligands for TNF.

\section{REFERENCES}

[1] M. Schoenberg, M. Weiss and P. Radermacher, "Outcome of Patients with Sepsis and Septic Shock after ICU Treatment," Langenbeck's Archives Surgery, Vol. 383, No. 1, 1998, pp. 44-48. doi:10.1007/s004230050090

[2] J. A. Kellum, L. Kong, M. P. Fink, L. A. Weissfield, D.
M. Yealy, M. R. Pinsky, J. Fine, A. Krichevsky, R. L. Delude and D. C. Angus, "Understanding the Inflammatory Cytokine Response in Pneumonia and Sepsis," Archives of International Medicine, Vol. 167, No. 15, 2007, pp. 1655-1663. doi:10.1001/archinte.167.15.1655

[3] J. Kellum, "Immunomodulation in Sepsis: The Role of Hemofiltration," Minerva Anestesiologica, Vol. 65, No. 6, 1999, pp. 410-418.

[4] J. A. Kellum and M. K. Dishart, "Effect of Hemofiltration Filter Adsorption on Circulating IL-6 Levels in Septic Rats," Critical Care, Vol. 6, No. 5, 2002, pp. 429-433. doi:10.1186/cc1528

[5] M. Song, J. Winchester, R. L. Albright, V. J. Capponi, M. D. Choquette and J. A. Kellum, "Cytokine Removal with a Novel Adsorbent Polymer," Blood Purification, Vol. 22, No. 5, 2004, pp. 428-434. doi:10.1159/000080235

[6] M. V. DiLeo, J. Kellum and W. J. Federspiel, "A Simple Mathematical Model of Cytokine Capture Using a Hemoadsorption Device," Annals of Biomedical Engineering, Vol. 37, No. 1, 2009, pp. 222-229. doi:10.1007/s10439-008-9587-8

[7] R. Stoltenburg, C. Reinemann and B. Strehlitz, "SELEXA (r)Evolutionary Method to Generate High-Affinity Nucleic Acid Ligands," Biomolecular Engineering, Vol. 24, No. 4, 2007, pp. 381-403. doi:10.1016/i.bioeng.2007.06.001

[8] S. J. Klug and M. Famulok, "All You Wanted to Know about SELEX," Molecular Biology Reports, Vol. 20, No. 2, 1994, pp. 97-107. doi:10.1007/BF00996358

[9] X. Yang and D. G. Gorenstein, "Progress in Thioaptamer Development," Current Drug Targets, Vol. 5, No. 8, 2004, pp. 705-715. doi:10.2174/1389450043345074

[10] J. W. Guthrie, C. L. A. Hamula, H. Zhang and X. C. Le, "Assays for Cytokines Using Aptamers," Methods, Vol. 38, No. 4, 2006, pp. 324-330. doi:10.1016/j.ymeth.2006.01.001

[11] Z. Zhang, X. Yan and C. Xu, "Oligonuecleotide Antagonist for Human Tumor Necrosis Factor A (TNF-a)," Institute for Viral Disease Control and Prevention Chinese Center for Disease Control and Prevention, United States, 2007.

[12] X. Yan, X. Gao and Z. Zhang, "Isolation and Characterization of 2'-Amino-Modified RNA Aptamers for Human TNFa," Genomics Proteomics \& Bioinformatics, Vol. 2, No. 1, 2004, pp. 32-42.

[13] N. Zammatteo, C. Girardeaux, D. Delforge, J.-J. Pireaux and J. Remacle, "Amination of Polystyrene Microwells: Application to the Covalent Grafting of DNA Probes for Hybridization Assays," Analytical Biochemistry, Vol. 236, No. 1, 1996, pp. 85-94. doi:10.1006/abio.1996.0135

[14] M. Matteucci and M. Caruthers, "Synthesis of Deoxyoligonucleotides on a Polymer Support," Journal of the American Chemical Society, Vol. 103, No. 11, 1981, pp. 31853191. doi:10.1021/ja00401a041

[15] L. S. Green, D. Jellinek, R. Jenison, A. Ostman, C.-H. Heldin and N. Janjic, "Inhibitory DNA Ligands to Platelet-Derived Growth Factor B-Chain," Biochemistry, Vol. 35, No. 45, 1996, pp. 14413-14424. doi:10.1021/bi961544+ 